KA-TP-02-2017

\title{
Quantum kinetic theory of a massless scalar model in the presence of a Schwarzschild black hole
}

\author{
Slava Emelyanov* \\ Institute for Theoretical Physics, \\ Karlsruhe Institute of Technology (KIT), \\ 76131 Karlsruhe, Germany
}

\begin{abstract}
We employ quantum kinetic theory to investigate local quantum physics in the background of spherically symmetric and neutral black holes formed through the gravitational collapse. For this purpose in mind, we derive and study the covariant Wigner distribution function $\mathcal{W}(x, p)$ near to and far away from the black-hole horizon. We find that the local density of the particle number is negative in the near-horizon region, while the entropy density is imaginary. These pose a question whether kinetic theory is applicable in the near-horizon region. We elaborate on that and propose a possible interpretation of how this result might nevertheless be self-consistently understood.
\end{abstract}

Keywords: quantum field theory, quantum kinetic theory, physics of black-hole evaporation

*Electronic address: viacheslav.emelyanov@kit.edu 


\section{INTRODUCTION}

The black-hole evaporation [1] is a remarkable discovery in the black-hole physics. This effect reveals itself in the positive flux of energy density being measurable sufficiently far away from the event horizon. The thermal profile of the mode distribution characterising this energy flux might imply that one could define other local observables which are usually attributable to normal/classical many-particle systems (rare gases, plasmas and so on). To our knowledge, there has been no progress in this direction. In this paper, we shall try to make it by exploiting quantum kinetic theory.

Our main purpose in this paper is therefore to study local kinetic state variables in the background of evaporating spherically symmetric black holes. A part of the local macroscopic variables correspond to the elements of the renormalised stress tensor $\left\langle\hat{T}_{\nu}^{\mu}(x)\right\rangle$ associated with a certain field model. These are the energy density, its flux and the pressure. In principle, these do not need any reference to the kinetic theory as being quantities directly computable from the first principles. However, there are many other variables which are not. These are the particle density $n(x)$ and the particle density flux $\mathbf{N}(x)$ as well as the local entropy density $s(x)$ and the entropy density flux $\mathbf{S}(x)$.

The framework within which we shall be working below is based on a massless scalar field conformally coupled to gravity. To do quantum kinetic theory, we need the scalar 2point function $W\left(x, x^{\prime}\right)$. It is in general a difficult problem to analytically derive $W\left(x, x^{\prime}\right)$ in Schwarzschild space. Nevertheless, if one employs the conformal symmetry of the scalar model, one can compute an approximate expression of the Wightman function close to and far away from the black-hole horizon [2]. However, the 2-point function is up to now unknown for physical black holes, i.e. those which have formed through the gravitational collapse. This is a technical problem we shall analytically address in this paper. The basic structure of $W\left(x, x^{\prime}\right)$ has been already conjectured by us in [3] (with further applications in [4] ) by exploiting the results of [2, 5]. We prove this conjecture in Sec. II and derive the higher-order corrections to that result as well. These corrections turn out to play a crucial role in re-obtaining the correct expression of the renormalised stress-energy tensor.

These preliminary steps will allow us to derive a covariant Wigner distribution $\mathcal{W}(x, p)$,

wherein $x$ and $p$ denote a space-time point and four-momentum (e.g., see [6]). We then apply this distribution for the derivation of the local macroscopic variables in the region far away and near to the black-hole horizon. Our results are presented in Sec. III. To sum it up, we find the standard picture far away from the event horizon, whereas its inapplicability in the near-horizon region as $n(x)$ and $s(x)$ turn out to be negative and imaginary, respectively.

We discuss our results in Sec. [V] and propose physical interpretation of how these can possibly be understood in a self-consistent manner.

Throughout this paper the fundamental constants are set to $c=G=k_{\mathrm{B}}=\hbar=1$, unless stated otherwise. We shall be employing a convention for the indices " 0 " and " 1 " to refer to the far-horizon region and the near-horizon region, respectively. The logic behind this 
notation is that $r_{H} / r \rightarrow 0$ and $r_{H} / r \sim 1$ hold far away from and near to the event horizon.

\section{SCALAR FIELD MODEL}

We shall be dealing with the scalar field $\Phi(x)$ in the background of Schwarzschild black hole of astrophysical mass $M$. We set the scalar-field mass to zero and assume the field is conformally coupled to gravity. The scalar Lagrangian is thus taken to be of the form

$$
\mathcal{L}=-\frac{1}{2} \Phi \square \Phi+\frac{1}{12} R \Phi^{2}
$$

where $R$ is the Ricci scalar which is, however, identically zero in the Schwarzschild geometry described by

$$
d s^{2}=g_{\mu \nu} d x^{\mu} d x^{\nu}=f(r) d t^{2}-\frac{d r^{2}}{f(r)}-r^{2} d \Omega^{2},
$$

where the lapse function $f(r) \equiv 1-r_{H} / r$ and $d \Omega$ is an element of the solid angle. The parameter $r_{H} \equiv 2 M$ is the Schwarzschild radius aka the size of the event horizon.

\section{A. Scalar Wightman function in Schwarzschild frame}

We have recently conjectured the structure of the Wightman 2-point function in the background of evaporating Schwarzschild black hole [3], wherein we have also used that to study the one-loop effects in QED in the far-from-horizon region and in a massless scalar model with the quartic self-interaction term in [4]. In this section, we shall prove that and also derive higher-order corrections with respect to $\Delta \mathbf{x}$ which have been neglected in [3].

It turns out that the approximate analytic expression of the scalar Wightman function $W\left(x, x^{\prime}\right)$ can be found with a comparably little computational effort if one takes advantage of the conformal symmetry of the scalar model. This observation allowed to compute the Wightman function for the Hartle-Hawking (HH) as well as Boulware state [2].

To derive the 2-point function for the physical black holes, we first perform a conformal transformation of the Schwarzschild metric to its ultra-static form $\bar{g}_{\mu \nu}(x)$, namely

$$
g_{\mu \nu}(x)=f(r) \bar{g}_{\mu \nu}(x)
$$

Correspondingly, the Wightman function $W\left(x, x^{\prime}\right)$ fulfilling $\square W\left(x, x^{\prime}\right)=0$ (we have taken

into account here that $R=0$ for the Schwarzschild black hole) can be written in the ultrastatic metric as follows

$$
W\left(x, x^{\prime}\right)=\bar{W}\left(x, x^{\prime}\right) /\left(f(r) f\left(r^{\prime}\right)\right)^{\frac{1}{2}},
$$

where $\bar{W}\left(x, x^{\prime}\right)$ satisfies

$$
\left(\bar{\square}-\frac{1}{6} \bar{R}\right) \bar{W}\left(x, x^{\prime}\right)=0 .
$$


According to our convention being extensively used below, all barred quantities are defined with respect to the ultra-static metric $\bar{g}_{\mu \nu}(x)$.

Since the Killing algebra of the space under consideration consists of the time translation as well as three generators of the rotational group, we look for a solution of (5) in the form

$$
\bar{W}\left(x, x^{\prime}\right)=\int_{\mathbf{R}} \frac{d \omega}{4 \pi \omega} e^{-i \omega\left(t-t^{\prime}\right)} \bar{K}_{\omega}\left(\mathbf{x}, \mathbf{x}^{\prime}\right)
$$

where the integral is over $\omega \in(-\infty,+\infty)$ and by definition

$$
\bar{K}_{\omega}\left(x, x^{\prime}\right) \equiv \frac{1}{4 \pi} \bar{\Delta}^{\frac{1}{2}}\left(\mathbf{x}, \mathbf{x}^{\prime}\right) \frac{\sin (\omega \rho)}{\omega \rho} \chi_{\omega}\left(\mathbf{x}, \mathbf{x}^{\prime}\right) \quad \text { with } \quad \rho \equiv\left(2 \bar{\sigma}\left(\mathbf{x}, \mathbf{x}^{\prime}\right)\right)^{\frac{1}{2}},
$$

where $\bar{\sigma}\left(\mathbf{x}, \mathbf{x}^{\prime}\right)$ is a geodetic interval for the spatial section of the ultra-static metric $\bar{g}_{\mu \nu}(x)$ and $\bar{\Delta}\left(\mathbf{x}, \mathbf{x}^{\prime}\right)$ the Van Vleck-Morette determinant.

Substituting (6) into (5), one obtains an equation which the unknown bi-scalar $\chi_{\omega}\left(\mathbf{x}, \mathbf{x}^{\prime}\right)$ must satisfy. Specifically, this reads

$$
\square \chi_{\omega}+\frac{1}{3}\left(\bar{R}_{i}^{j}-2 \omega^{2} \delta_{i}^{j}\right) \bar{\sigma}^{i} \bar{\nabla}_{j} \chi_{\omega}-\frac{1}{12}\left(2 \bar{R}_{i k ; j}-\bar{R}_{i j ; k}\right) \bar{\sigma}^{i} \bar{\sigma}^{j} \bar{\nabla}^{k} \chi_{\omega}+\mathrm{O}\left(\left(\bar{\sigma}^{i}\right)^{3}\right)=0,
$$

where $\bar{\sigma}^{i} \equiv \bar{\nabla}^{i} \bar{\sigma}$ and $i, j$ run from 1 to 3. In the derivation of Eq. (8) we have employed the fact that $\bar{R}_{; i}-2 \bar{R}_{i ; j}^{j}$ identically vanishes for any lapse function $f(r)$ and

$$
9 \bar{R}_{; i j}+9 \bar{R}_{i j ; k}^{; k}-24 \bar{R}_{i k ; j}^{; k}-12 \bar{R}_{i k} \bar{R}_{j}^{k}+6 \bar{R}^{k n} \bar{R}_{i k j n}+4 \bar{R}_{i k n m}\left(\bar{R}_{j}^{m n k}+\bar{R}_{j}^{k n m}\right)=0
$$

for the lapse function of the form $1-r_{H} / r+\Lambda r^{2} / 3$. It means that the equation (8) is applicable to a wide class of static spacetimes. As a consequence, we have

$$
\bar{\square} \bar{\Delta}^{\frac{1}{2}}\left(\mathbf{x}, \mathbf{x}^{\prime}\right)=\frac{1}{6} \bar{R}(\mathbf{x}) \bar{\Delta}^{\frac{1}{2}}\left(\mathbf{x}, \mathbf{x}^{\prime}\right)+\mathrm{O}\left(\left(\bar{\sigma}^{i}\right)^{3}\right) .
$$

We need now to solve Eq. (8) to eventually obtain the 2-point function. In the leading order of the approximation, the bi-scalar $\chi_{\omega}\left(x, x^{\prime}\right)$ reads

$$
\chi_{\omega}\left(\mathbf{x}, \mathbf{x}^{\prime}\right)=a_{\omega}+b_{\omega} \frac{\left(f(r) f\left(r^{\prime}\right)\right)^{\frac{1}{2}}}{r r^{\prime}}\left(1 \pm i \omega \Delta r_{\star}+\alpha_{\omega}\left(r, r^{\prime}\right) \Delta r_{\star}^{2}+\beta_{\omega}\left(r, r^{\prime}\right) \bar{\sigma}\left(\mathbf{x}, \mathbf{x}^{\prime}\right)\right),
$$

where $\Delta r_{\star} \equiv r_{\star}-r_{\star}^{\prime}$ with $r_{\star}$ denoting the Regge-Wheeler radial coordinate and

$$
\begin{aligned}
& \alpha_{\omega}\left(r, r^{\prime}\right) \approx-\frac{\omega^{2}}{2}, \\
& \beta_{\omega}\left(r, r^{\prime}\right) \approx+\frac{\omega^{2}}{3}+\frac{r_{H}}{12\left(r r^{\prime}\right)^{\frac{3}{2}}}+\frac{r_{H}}{4\left(r r^{\prime}\right)^{\frac{3}{2}}}\left(f(r) f\left(r^{\prime}\right)\right)^{\frac{1}{2}} .
\end{aligned}
$$

The sign in front of the second term in the parenthesis of Eq. (11) cannot be fixed without referring to the mode expansion of the scalar field. We take it negative in the far-horizon region and positive in the near-horizon region, because then $\left\langle\hat{T}_{t}^{r}\right\rangle$ has a correct sign.

The bi-scalar $\chi_{\omega}\left(\mathbf{x}, \mathbf{x}^{\prime}\right)$ given in (11) with (12) is a solution of the equation $\square \chi_{\omega}\left(\mathbf{x}, \mathbf{x}^{\prime}\right)=0$ up to the order of $\mathrm{O}\left(\mathbf{x}-\mathbf{x}^{\prime}\right)$. There are infinitely many solutions of this type. However, we 
shall show below that the bi-functions defined in Eq. (12) yield the stress-energy tensor of the scalar field as found in [5, 7]. ${ }^{1}$

We now need to determine the functions $a_{\omega}$ and $b_{\omega}$. With this purpose in mind, we consider the far-horizon $\left(r, r^{\prime} \gg r_{H}\right)$ and near-horizon $\left(r, r^{\prime} \sim r_{H}\right)$ region separately.

\section{Far-horizon region}

One might expect from the physical grounds that the Wightman function $W\left(x, x^{\prime}\right)$ must reduce to the Minkowski 2-point function, $W_{M}\left(x, x^{\prime}\right)$, in the asymptotically flat region, i.e. $W\left(x, x^{\prime}\right) \rightarrow W_{M}\left(x, x^{\prime}\right)$ in the limit $r \rightarrow \infty$ with $\left|r-r^{\prime}\right| \ll R$, where $R$ is the distance to the black-hole centre here and below. Indeed, if it had not be the case, then it would be not legitimate to use the Minkowski-space approximation in describing and testing particle physics in the colliders on earth. This implies

$$
a_{\omega, 0} \approx+4 \omega^{2} \theta(+\omega),
$$

where $\theta(z)$ is the Heaviside step function.

The function $b_{\omega, 0}$ cannot be so simply determined. However, if we set

$$
b_{\omega, 0} \approx+27(\omega M)^{2} n_{\beta}(\omega)\left(\theta(\omega)+e^{\beta \omega} \theta(-\omega)\right) \quad \text { with } \quad n_{\beta}(\omega) \equiv 1 /\left(e^{\beta \omega}-1\right),
$$

where $\beta=2 \pi / \kappa \equiv 8 \pi M$ is the inverse Hawking temperature $T_{H} \equiv 1 / 8 \pi M[1]$, then we obtain our previous result [3, 4] (with $\chi_{\omega}\left(\mathbf{x}, \mathbf{x}^{\prime}\right)$ in the limit $\mathbf{x}^{\prime} \rightarrow \mathbf{x}$ ) which is in agreement with [2, 5]. Specifically, we find

$$
W_{0}\left(x, x^{\prime}\right)=W_{M}\left(x, x^{\prime}\right)+\Delta W_{0}\left(x, x^{\prime}\right),
$$

where the first term is the Minkowski 2-point function, i.e.

$$
W_{M}\left(x, x^{\prime}\right) \approx \int \frac{d^{3} \mathbf{k}}{(2 \pi)^{3}} \frac{1}{2 k_{0}} \exp (-i k \Delta x) \text { with } \quad k_{0}=|\mathbf{k}|,
$$

and the higher-order correction to $W_{M}\left(x, x^{\prime}\right)$ reads

$$
\begin{aligned}
\Delta W_{0}\left(x, x^{\prime}\right) \approx \frac{27 r_{H}^{2}}{16 R^{2}} \int & \frac{d^{3} \mathbf{k}}{(2 \pi)^{3}} \frac{n_{\beta}\left(k_{0}\right)}{k_{0}} \cos (\mathbf{k} \Delta \mathbf{x}) \\
& \times\left[\left(1-\frac{k_{0}^{2}}{6}\left(3 \Delta r^{2}-\Delta \mathbf{x}^{2}\right)\right) \cos \left(k_{0} \Delta t\right)+k_{0} \Delta r \sin \left(k_{0} \Delta t\right)\right],
\end{aligned}
$$

where $k \Delta x \equiv k_{\mu} \Delta x^{\mu}$ and $\Delta \mathrm{x}^{2} \equiv \Delta x^{2}+\Delta y^{2}+\Delta z^{2}$ by our convention. The coordinates $x, y$ and $z$ here are local Cartesian coordinates introduced at the distance $R$ from the centre of the black hole.

\footnotetext{
1 There are extra terms in $\alpha_{\omega}\left(r, r^{\prime}\right)$ and $\beta_{\omega}\left(r, r^{\prime}\right)$ which give sub-leading contributions to the diagonal elements of $\left\langle\hat{T}_{\nu}^{\nu}\right\rangle$ in both far-horizon and near-horizon region. For instance, there are additional terms vanishing as $f(r)$ near the horizon which we have omitted. We shall study these in detail elsewhere.
} 
The 2-point function (15) is more general than that we have found in [3, 4] as it also contains the higher-order corrections in $\Delta \mathbf{x}$. We shall show below that $\Delta W_{0}\left(x, x^{\prime}\right)$ yields the correct expression of the renormalised stress tensor $\left\langle\hat{T}_{\nu}^{\mu}\right\rangle$ at the spatial infinity.

\section{Near-horizon region}

One might expect from the equivalence principle that the Wightman function $W\left(x, x^{\prime}\right)$ in the near-horizon region $r, r^{\prime} \sim r_{H}$ with $\left|r-r^{\prime}\right| \ll r_{H}$ must approximately be given by the 2-point function $W_{M}\left(x, x^{\prime}\right)$ as in Minkowski space when expressed in the local inertial coordinates. We take this for granted below to fix the function $a_{\omega, 1}$.

To determine $a_{\omega, 1}$, one needs first to introduce new spatial coordinates instead of the angle coordinates in the following manner:

$$
y^{2}+z^{2}=4 r_{H}^{2} \tan ^{2}(\theta / 2) \text { and } z / y=\tan \phi \quad \text { with } y^{2}+z^{2} \ll r_{H}^{2} .
$$

The geodetic distance $\sigma\left(x, x^{\prime}\right)$ in these coordinates acquires a comparably simple structure, namely

$$
\sigma\left(x, x^{\prime}\right) \approx \frac{1}{\kappa^{2}}\left(f(r) f\left(r^{\prime}\right)\right)^{\frac{1}{2}}\left(\cosh \kappa \Delta t-\frac{f(r)+f\left(r^{\prime}\right)+\kappa^{2}\left(\Delta y^{2}+\Delta z^{2}\right)}{2\left(f(r) f\left(r^{\prime}\right)\right)^{\frac{1}{2}}}\right),
$$

where we have neglected the higher-order corrections and $\kappa \equiv 1 / 2 r_{H}$ by definition. It is worth emphasising that $\sigma\left(x, x^{\prime}\right)$ has been computed directly as a geometrical quantity in Schwarzschild space. Having calculated the geodetic distance $\bar{\sigma}\left(\mathbf{x}, \mathbf{x}^{\prime}\right)$ of the spatial section of the ultra-static metric $\bar{g}_{\mu \nu}(x)=g_{\mu \nu}(x) / f(r)$ in the near-horizon region, we then obtain

$$
\sigma\left(x, x^{\prime}\right) \approx \frac{1}{\kappa^{2}}\left(f(r) f\left(r^{\prime}\right)\right)^{\frac{1}{2}}(\cosh \kappa \Delta t-\cosh \kappa \rho)
$$

where $\rho \equiv\left(2 \bar{\sigma}\left(\mathbf{x}, \mathbf{x}^{\prime}\right)\right)^{\frac{1}{2}}$ as defined in Eq. (17). Therefore, in order to have $W\left(x, x^{\prime}\right)$ be approximately equal to $-1 /\left(8 \pi^{2} \sigma\left(x, x^{\prime}\right)\right)$ in the near-horizon region, one must set

$$
a_{\omega, 1} \approx+4 \omega^{2} n_{\beta}(\omega) e^{\beta \omega}(\theta(+\omega)+\theta(-\omega)) .
$$

In order to determine the function $b_{\omega, 1}$, one needs to treat the mode expansion of the scalar field operator $\hat{\Phi}(x)$. Employing the results of [2, 5], we obtain

$$
b_{\omega, 1} \approx-27(\omega M)^{2} n_{\beta}(\omega)\left(\theta(+\omega)+e^{\beta \omega} \theta(-\omega)\right) .
$$

Thus, the 2-point function near the horizon reads

$$
W_{1}\left(x, x^{\prime}\right)=W_{M}\left(x, x^{\prime}\right)+\Delta W_{1}\left(x, x^{\prime}\right),
$$

where $W_{M}\left(x, x^{\prime}\right) \approx W_{\mathrm{HH}}\left(x, x^{\prime}\right)$ for $r, r^{\prime} \rightarrow r_{H}$ and

$$
W_{\mathrm{HH}}\left(x, x^{\prime}\right) \approx \frac{\bar{\Delta}^{\frac{1}{2}}\left(\mathbf{x}, \mathbf{x}^{\prime}\right)}{\left(f(r) f\left(r^{\prime}\right)\right)^{\frac{1}{2}}} \int \frac{\omega d \omega}{(2 \pi)^{2}} n_{\beta}(\omega) e^{\beta \omega}\left[e^{-i \omega \Delta t}+e^{-\beta \omega} e^{+i \omega \Delta t}\right] \frac{\sin \omega \rho}{\omega \rho}
$$


with $\omega>0$, and the correction to $W_{M}\left(x, x^{\prime}\right)$ reads

$$
\begin{aligned}
\Delta W_{1}\left(x, x^{\prime}\right) \approx & -\frac{27}{16} \bar{\Delta}^{\frac{1}{2}}\left(\mathbf{x}, \mathbf{x}^{\prime}\right) \int \frac{\omega d \omega}{(2 \pi)^{2}} n_{\beta}(\omega) \frac{\sin \omega \rho}{\omega \rho} \\
& \times\left[\left(1-\frac{\omega^{2}}{2} \Delta r_{\star}^{2}+\frac{1}{3}\left(\omega^{2}+\kappa^{2}\right) \bar{\sigma}\left(\mathbf{x}, \mathbf{x}^{\prime}\right)\right) \cos (\omega \Delta t)-\omega \Delta r_{\star} \sin (\omega \Delta t)\right] .
\end{aligned}
$$

The 2-point function $W_{1}\left(x, x^{\prime}\right)$ is also more general than that we have found in [3, 4] in the near-horizon region, but reduces to it if one takes the limit $\mathbf{x}^{\prime} \rightarrow \mathbf{x}$ in the square brackets.

\section{B. Scalar Wightman function in Fermi frame}

For the applications below, we need to express the scalar 2-point function $W_{1}\left(x, x^{\prime}\right)$ via the Fermi normal coordinates. These coordinates are characterised by a geodesic with the tangent vector $G$ [8].

One can introduce an orthonormal tetrad $e_{a}^{\mu}=\left(e_{t_{F}}^{\mu}, e_{x_{F}}^{\mu}, e_{y_{F}}^{\mu}, e_{z_{F}}^{\mu}\right)$, such that $e_{t_{F}}^{\mu}$ is a (time-like) tangent vector to the geodesic $G$ describing a radial free fall towards the black hole. This tetrad is given by

$$
\begin{aligned}
e_{t_{F}}^{\mu} \partial_{\mu} & =\frac{1}{f(r)} \partial_{t}-(1-f(r))^{\frac{1}{2}} \partial_{r}, \\
e_{x_{F}}^{\mu} \partial_{\mu} & =-\frac{(1-f(r))^{\frac{1}{2}}}{f(r)} \partial_{t}+\partial_{r}, \\
e_{y_{F}}^{\mu} \partial_{\mu} & =\frac{1}{r} \partial_{\theta}, \quad e_{z_{F}}^{\mu} \partial_{\mu}=\frac{1}{r \sin \theta} \partial_{\phi} .
\end{aligned}
$$

In the Fermi normal coordinates $x^{a}=\left(t_{F}, x_{F}, y_{F}, z_{F}\right)$, the metric tensor along the geodesic has the Minkowski form, i.e. $\left.g_{a b}\right|_{G}=\eta_{a b}$, such that $\left.\Gamma_{a b}^{c}\right|_{G}=0$. It is worth noticing that the Fermi time $t_{F}$ is identical to the Painlevé-Gullstrand time $\tau$ we have made use of in [4]. For space-time points close to the geodesic $G$, one has

$$
g_{a b}\left(x_{F}\right)=\eta_{a b}-\kappa_{a b} R_{a c b d}\left(x_{F}\right) x_{F}^{c} x_{F}^{d}+\mathrm{O}\left(x_{F}^{3}\right),
$$

as shown in [8], where there is no summation over $a$ and $b$ in the second term of (27) and

$$
\kappa_{a b}=\frac{1}{2}\left(\delta_{a}^{0}+\delta_{b}^{0}+\frac{1}{3} \sum_{i=1}^{3}\left(\delta_{a}^{i}+\delta_{b}^{i}\right)\right) .
$$

The geodetic distance $\sigma\left(x, x^{\prime}\right)$ in these coordinates is given by

$$
\sigma\left(x, x^{\prime}\right)=\sigma\left(x_{F}, x_{F}^{\prime}\right)=\frac{1}{2} \eta_{a b}\left(x_{F}-x_{F}^{\prime}\right)^{a}\left(x_{F}-x_{F}^{\prime}\right)^{b}+\mathrm{O}\left(\frac{r_{H} \Delta x_{F}^{4}}{R^{3}}\right) .
$$

where we have taken one of the points on the geodesic.

In the local inertial frame associated with the geodesic $G$, the Wightman function $W\left(x, x^{\prime}\right)$ should naturally be given by $W_{M}\left(x, x^{\prime}\right)$ whenever it is legitimate to neglect geometrical 
corrections. This is exactly what we have obtained in Eq. (15) and Eq. (23). This means no significant (quantum) effect can be discovered in the near-horizon region for an observer freely falling in the black hole of a sufficiently large mass $M .^{2}$

Far away from the black-hole horizon, the Schwarzschild coordinates $x$ go over to the Minkowski coordinates $x_{M}$. Therefore, the 2-point function $W_{0}\left(x, x^{\prime}\right)$ is already given in the flat coordinates for $R \gg r_{H}$. In the region near to the black-hole horizon, the Schwarzschild coordinates $x$ considerably differ from the Fermi ones $x_{F}$. Having computed $x_{F}$ as functions of $x$ from (26), we obtain

$$
W_{1}\left(x_{F}, x_{F}^{\prime}\right)=W_{M}\left(x_{F}, x_{F}^{\prime}\right)+\Delta W_{1}\left(x_{F}, x_{F}^{\prime}\right),
$$

where

$$
\begin{aligned}
\Delta W_{1}\left(x_{F}, x_{F}^{\prime}\right) \approx-\frac{27}{4} \int & \frac{d^{3} \mathbf{k}}{(2 \pi)^{3}} \frac{n_{2 \beta}\left(k_{0}\right)}{k_{0}} \cos \left(\mathbf{k} \Delta \mathbf{x}_{F}\right) \\
\times & {\left[\left(1-\frac{k_{0}^{2}}{6}\left(3 \Delta x_{F}^{2}-\Delta \mathbf{x}_{F}^{2}\right)\right) \cos \left(k_{0} \Delta t_{F}\right)-k_{0} \Delta x_{F} \sin \left(k_{0} \Delta t_{F}\right)\right], }
\end{aligned}
$$

where $\mathbf{x}_{F}=\left(x_{F}, y_{F}, z_{F}\right)$ by definition. It should be mentioned that we have explicitly checked that $\Delta W_{1}\left(x_{F}, x_{F}^{\prime}\right)$ coincides with $\Delta W_{1}\left(x, x^{\prime}\right)$ given in Eq. (25) up to the order of $\Delta x_{F}^{2}$ including. Therefore, the above expression of $\Delta W_{1}\left(x_{F}, x_{F}^{\prime}\right)$ should be reliable up to this order only. The same holds for the 2-point function $W_{0}\left(x, x^{\prime}\right)$ far away from the black hole. The reason of this limitation comes from the bi-scalar $\chi_{\omega}\left(\mathbf{x}, \mathbf{x}^{\prime}\right)$ which we have determined only up to that order. This approximation is, however, adequate for our purposes below.

\section{QUANTUM KINETIC APPROACH TO BLACK-HOLE PHYSICS}

\section{A. Relativistic kinetic theory: Brief introduction}

Many-particle systems can be described with the aid of the local macroscopic state variables. These variables are the particle density number, energy density, pressure and so on. In the framework of the kinetic theory, these are defined through the one-particle distribution function. This distribution is usually denoted by $f_{\mathrm{cl}}(x, p)$, where $x^{\mu}=(t, \mathbf{x})$ and $p^{\mu}=\left(p_{0}, \mathbf{p}\right)$ with $g_{\mu \nu} p^{\mu} p^{\nu}=m^{2}$ are a space-time coordinate and momentum coordinate, respectively.

The space-time evolution of the distribution function $f_{\mathrm{cl}}(x, p)$ is governed by the transport equation, which is a relativistic generalisation of the famous Boltzmann equation. Specifically, this reads

$$
\left(p^{\mu} \frac{\partial}{\partial x^{\mu}}-\Gamma_{\mu \nu}^{\lambda} p^{\mu} p^{\nu} \frac{\partial}{\partial p^{\lambda}}\right) f_{\mathrm{cl}}(x, p)=C\left[f_{\mathrm{cl}}(x, p)\right]
$$

\footnotetext{
${ }^{2}$ For black holes of mass in the range $10^{10} \mathrm{~g} \lesssim M \ll 10^{16} \mathrm{~g}$, there are certain tiny effects (the modification of the light deflection angle and Debye-like screening of a point-like charge) which might be testable [3, 10] assuming these exist in nature and their number density is sufficiently large.
} 
in curved spacetime, where $C\left[f_{\mathrm{cl}}(x, p)\right]$ is a collision integral taking into account binary scattering processes of the constituent particles of the system, and no external field apart from gravity has been assumed (e.g., see [11]).

The main state variables are the particle four-current, i.e.

$$
N^{\mu}(x)=(-g)^{\frac{1}{2}} \int \frac{d^{3} \mathbf{p}}{p_{0}} p^{\mu} f_{\mathrm{cl}}(x, p),
$$

the energy-momentum tensor reading

$$
T^{\mu \nu}(x)=(-g)^{\frac{1}{2}} \int \frac{d^{3} \mathbf{p}}{p_{0}} p^{\mu} p^{\nu} f_{\mathrm{cl}}(x, p),
$$

and the entropy four-flow defined as

$$
S^{\mu}(x)=-(-g)^{\frac{1}{2}} \int \frac{d^{3} \mathbf{p}}{p_{0}} p^{\mu} f_{\mathrm{cl}}(x, p)\left(\ln \left(h^{3} f_{\mathrm{cl}}(x, p)\right)-1\right) .
$$

These macroscopic variables are local. This fact allows to describe equilibrium as well as non-equilibrium macroscopic states of the system by these variables.

The macroscopic conservation laws

$$
\begin{aligned}
& \nabla_{\mu} N^{\mu}(x)=0, \\
& \nabla_{\mu} T^{\mu \nu}(x)=0 .
\end{aligned}
$$

can be shown to hold as a consequence of the transport equation (32) and the microscopic conservation laws of the particle number as well as the four-momentum. These can in turn be used to derive the well-known hydrodynamic equations like the continuity equation or the Euler equations with the relativistic corrections.

In the kinetic theory, one can also prove the Boltzmann $H$-theorem. This theorem states that the entropy production rate $\sigma(x)$ at any space-time point is never negative, i.e.

$$
\sigma(x) \equiv \nabla_{\mu} S^{\mu}(x) \geq 0 .
$$

For the sake of completeness, we want finally to remind about the role of the hydrodynamic (e.g., Eckart or Landau-Lifshitz) velocity $U^{\mu}(x)$. This allows to define covariant state variables corresponding to the particle number density $N^{\mu} U_{\mu}$, the energy density $T_{\mu \nu} U^{\mu} U^{\nu}$ and so on. For more details, we refer to the references [6, 11].

\section{B. Covariant Wigner distribution $\mathcal{W}(x, p)$}

Starting with local quantum field theory, one can introduce the distribution function and the transport equation associated with it. This leads to quantum kinetic theory [6]. The main object in this theory is the Wigner operator

$$
\hat{\mathcal{W}}(x, p) \equiv \frac{4 \pi}{(2 \pi)^{5}} \int d^{4} y e^{-i p y} \hat{\Phi}(x+y / 2) \hat{\Phi}(x-y / 2)
$$


Note that we are working in the Fermi coordinate frame here. It allows us to employ the ordinary Fourier transform as if the Fermi frame is infinitely large. This does not serve any problem whenever the physics we are interested in is characterised by a length scale being much smaller than a characteristic curvature scale.

Once we have a quantum system described by a certain state, we can relate with it the Wigner distribution

$$
\mathcal{W}(x, p)=\langle\hat{\mathcal{W}}(x, p)\rangle .
$$

It is worth pointing out that there is no direct probabilistic interpretation of the covariant Wigner distribution in terms of particles [6]. This can be understood from its very definition which is entirely independent on the covariant wave function. This is in contrast to the classical one-particle distribution $f_{\mathrm{cl}}(x, p)$ introduced above, which is a probability density giving a number of particles in a spatial volume $\Delta \mathrm{x}^{3}$ with the momentum in the interval between $\mathbf{p}$ and $\mathbf{p}+\Delta \mathbf{p}$. Despite of the lack of the straightforward physical meaning of $\mathcal{W}(x, p)$ in terms of particles, we want to employ $\mathcal{W}(x, p)$ in Schwarzschild geometry in order to get further insights about black holes and physics related to their evaporation.

\section{Wigner distribution in presence of evaporating black hole}

We now derive the covariant Wigner distribution associated with the scalar model in the background of the black hole formed via the gravitational collapse.

\section{Far-horizon region}

Far away from the black-hole horizon $R \gg r_{H}$, the 2-point function is given by (15). The contribution to the Wigner distribution (38) comes from only those terms in $W_{0}\left(x, x^{\prime}\right)$ which contain the positive frequency modes. The result reads

$$
\mathcal{W}_{0}(x, p) \approx \frac{3^{3} M^{2} n_{\beta}\left(p_{0}\right)}{2^{5} \pi^{3} R^{2} p_{0}}\left[1-p_{0} P_{1}\left(\frac{\mathbf{n p}}{p}\right) \partial_{p}+\frac{p_{0}^{2}}{3 p} P_{2}\left(\frac{\mathbf{n p}}{p}\right)\left(p \partial_{p}^{2}-\partial_{p}\right)\right] \delta\left(p_{0}-p\right),
$$

where $P_{n}(z)$ is the Legendre polynomial of the order $n \in \mathbb{N}_{0}$ and

$$
p \equiv|\mathbf{p}| \text { and } \quad \mathbf{n} \equiv \mathbf{R} / R .
$$

In deriving the Wigner distribution $\mathcal{W}_{0}(x, p)$, we have omitted terms which vanish faster than $1 / R^{2}$ in the limit $R \rightarrow \infty$.

The prefactors of the first, second and third term in $\mathcal{W}_{0}(x, p)$ are proportional to the Legendre polynomial of the zeroth, first and second order, respectively. This resembles a similar structure of the monopole, dipole and quadrupole potentials in the multiple expansion of the electrostatic potential performed sufficiently far away from a gas of charged particles. Therefore, we shall occasionally refer to these terms in the following as to the monopole-, dipole- and quadrupole-like term, respectively. 


\section{Near-horizon region}

In the near-horizon region $R \sim r_{H}$, the 2-point function has the form (23). Substituting this into (38), we obtain

$$
\mathcal{W}_{1}(x, p) \approx-\frac{3^{3} n_{2 \beta}\left(p_{0}\right)}{2^{5} \pi^{3} p_{0}}\left[1+p_{0} P_{1}\left(\frac{\mathbf{m p}}{p}\right) \partial_{p}+\frac{p_{0}^{2}}{3 p} P_{2}\left(\frac{\mathbf{m p}}{p}\right)\left(p \partial_{p}^{2}-\partial_{p}\right)\right] \delta\left(p_{0}-p\right),
$$

where we have taken into account the condition $p_{0}>0$ and

$$
\mathbf{m} \equiv(1,0,0)
$$

in the Fermi coordinate frame. Note that the structure of $\mathcal{W}_{1}(x, p)$ is the same as that of $\mathcal{W}_{0}(x, p)$ up to the total prefactor and the sign of the dipole-like term.

The distribution $\mathcal{W}_{1}(x, p)$ does not contain a contribution from $W_{M}\left(x_{F}, x_{F}^{\prime}\right) \approx W_{\mathrm{HH}}\left(x, x^{\prime}\right)$ which is an important part of the total 2-point function $W_{1}\left(x, x^{\prime}\right)$ as it provides the proper singularity structure for the Feynman propagator. The reason is that it has no modes with the positive frequency. ${ }^{3}$ This is reasonable as the Wigner distribution of the vacuum 2-point function in Minkowski space is trivial as well.

However, there is a non-vanishing contribution of $W_{M}\left(x, x^{\prime}\right)$ to the renormalised stress tensor if we approximate it by $W_{\mathrm{HH}}\left(x, x^{\prime}\right){ }^{4}$ The renormalisation is performed by employing the point-splitting technique and making the substitution

$$
W_{\mathrm{HH}}\left(x, x^{\prime}\right) \rightarrow W_{\mathrm{HH}}\left(x, x^{\prime}\right)-H\left(x, x^{\prime}\right)
$$

where $H\left(x, x^{\prime}\right)$ is the Hadamard parametrix (e.g., see [12, 13]). The parametrix serves to cancel the singular part of $W_{\mathrm{HH}}\left(x, x^{\prime}\right)$ in the coincidence limit $x^{\prime} \rightarrow x$. It also provides extra non-vanishing (geometrical) terms in $\left\langle T_{\nu}^{\mu}\right\rangle$ including the trace aka conformal anomaly. Specifically, it was found in [2] that

$$
\left\langle T_{b}^{a}\right\rangle_{\mathrm{HH}} \approx-\frac{\kappa^{4}}{120 \pi^{2}} \operatorname{diag}(3,3,1,1) \text { for } \quad R \sim r_{H}
$$

This result for $\left\langle T_{\nu}^{\mu}\right\rangle_{\mathrm{HH}}$ is close to that obtained by the numerical calculations [5, 14]. This implies that the prefactor in front of the parameter $a_{\omega}$ appearing in the bi-scalar $\chi_{\omega}\left(\mathbf{x}, \mathbf{x}^{\prime}\right)$ has actually to depend on the spatial coordinates and asymptotically approach 1 as faster as $1 / R^{4}$ for $R \rightarrow \infty$.

To sum it up, the distribution $\mathcal{W}_{1}(x, p)$ is expected to give the stress tensor renormalised as in [5], i.e. the relative part of the total energy-momentum tensor $\left\langle T_{\nu}^{\mu}\right\rangle$ with respect to $\left\langle T_{\nu}^{\mu}\right\rangle_{M}$. This seems to be reasonable as only this part provides the crucial term resulting in the black-hole evaporation and, hence, related to the Hawking particles.

\footnotetext{
${ }^{3}$ Thus, this will contribute if we extend the allowed values of $p_{0}$ from $-\infty$ to $+\infty$ in the formula (38), but the physics in terms of particles then becomes obscure.

${ }^{4}$ Strictly speaking, $W_{M}\left(x, x^{\prime}\right)$ and $W_{\mathrm{HH}}\left(x, x^{\prime}\right)$ are not equal to each other near horizon in the Schwarzschild frame, unless one sets $f^{\prime}(r) / 2=\kappa$ in $W_{M}\left(x, x^{\prime}\right)$ for $r \sim r_{H}$. This subtlety originates from the coordinate transformation to the local inertial frame near horizon and is inessential for the derivation of $\mathcal{W}_{1}(x, p)$.
} 


\section{Energy-momentum tensor $\left\langle\hat{T}_{\mu \nu}(x)\right\rangle$}

Having the distribution function $\mathcal{W}(x, p)$, we are able to compute the energy-momentum tensor $\left\langle\hat{T}_{\mu \nu}\right\rangle$ as its second moment with respect to the momentum $p_{\mu}$, namely

$$
\left\langle\hat{T}_{\mu \nu}(x)\right\rangle=\int d^{4} p p_{\mu} p_{\nu}\langle\hat{\mathcal{W}}(x, p)\rangle
$$

where the integration over $p_{0}$ is in the interval $(0,+\infty)$.

\section{Far-horizon region}

Employing our result for the Wigner distribution far away from the black hole, we obtain

$$
\left\langle\hat{T}_{\nu}^{\mu}\right\rangle \approx \frac{1}{4 \pi R^{2}} \int \frac{d p_{0}}{2 \pi} \frac{p_{0} \Gamma_{p_{0}}}{e^{\beta p_{0}}-1}\left[\begin{array}{cc}
+1 & +1 \\
-1 & -1
\end{array}\right] \text { for } R \gg r_{H},
$$

where the indices $\mu, \nu$ run over $\{t, r\}$ and the rest elements of $\left\langle\hat{T}_{\nu}^{\mu}\right\rangle$ vanish. We have introduced $\Gamma_{p_{0}}=27\left(p_{0} M\right)^{2}$ which corresponds to the DeWitt approximation that we have been employing throughout this paper. It is worth pointing out that the $t$-component of $\left\langle\hat{T}_{\nu}^{\mu}\right\rangle$ is due to the monopole-like term in (40), whereas its non-diagonal elements come from the dipole-like term in $\mathcal{W}_{0}(x, p)$ and the $r r$-component of the stress tensor originates from the monopole- and quadrupole-like term of the Wigner distribution. This result for the stress tensor $\left\langle\hat{T}_{\nu}^{\mu}\right\rangle$ given above is consistent with [5, 7] and can also be directly obtained by using, e.g., the point-splitting technique (see Appendix $\mathrm{A}$ for some details).

It is a well-known fact that the energy density far away from the black hole is positive, i.e. $\left\langle\hat{T}_{t}^{t}\right\rangle>0$, as well as its flux in the radial direction is also positive, i.e. $\left\langle\hat{T}_{t}^{r}\right\rangle>0$. It implies that there is a positive energy flux from the black hole. Thus, we re-derive this Hawking's discovery by use of the quantum kinetic approach.

It is tempting to define an effective Wigner distribution as follows

$$
\begin{aligned}
\mathcal{W}_{\mathrm{eff}, 0}(x, p) & =\frac{\Gamma_{p_{0}}}{32 \pi^{3} p_{0}^{3} R^{2}} n_{\beta}\left(p_{0}\right) \sum_{l=0}^{2}(2 l+1) P_{l}\left(\frac{\mathbf{n p}}{p}\right) \delta\left(p_{0}-p\right) \\
& \approx \frac{1}{8 \pi^{2}} \frac{\Gamma_{p_{0}}}{p_{0}^{3} R^{2}} n_{\beta}\left(p_{0}\right) \delta\left(p_{\theta}\right) \delta\left(p_{\phi}\right) \delta\left(p_{0}-p\right)
\end{aligned}
$$

where we have extended the finite summation over $l$ to the infinity and used a sum representation of the delta function in terms of the spherical harmonics. Furthermore, we may define an effective one-particle distribution

$$
f_{\mathrm{eff}, 0}(x, p)=\frac{1}{8 \pi^{2}} \frac{\Gamma_{p_{0}}}{p_{0}^{2} R^{2}} \frac{1}{e^{\beta p_{0}}-1} \delta\left(p^{\theta}\right) \delta\left(p^{\phi}\right),
$$

which has already been introduced in [4] with slightly different notations. We shall demonstrate below its usefulness. 


\section{Near-horizon region}

Substituting $\mathcal{W}_{1}(x, p)$ into Eq. (46), we obtain

$$
\left\langle\hat{T}_{b}^{a}\right\rangle \approx \frac{1}{\pi r_{H}^{2}} \int \frac{d p_{0}}{2 \pi} \frac{p_{0} \Gamma_{p_{0}}}{e^{2 \beta p_{0}}-1}\left[\begin{array}{l}
-1+1 \\
-1+1
\end{array}\right] \text { for } \quad R \sim r_{H}
$$

where $a, b$ run over $\left\{t_{F}, x_{F}\right\}$ and the rest elements of $\left\langle\hat{T}_{b}^{a}\right\rangle$ are suppressed in the Schwarzschild frame (see Appendix $\mathrm{A}$ for further details).

The energy density $\left\langle\hat{T}_{t_{F}}^{t_{F}}\right\rangle$ is negative near the horizon, whereas $\left\langle\hat{T}_{t_{F}}^{x_{F}}\right\rangle$ is positive. This physically implies that there is a flux of negative energy towards the black hole. The change of the energy flux direction well away from the black-hole horizon was first found in [15] with the physical insight that the vacuum spacetime itself is unstable at the quantum level. The same observation has been recently made in [16].

Analogous to the far-horizon region, one can introduce an effective Wigner distribution and associated with it an effective one-particle distribution function, namely

$$
f_{\mathrm{eff}, 1}(x, p)=-\frac{1}{2 \pi^{2} r_{H}^{2}} \frac{\Gamma_{p_{0}}}{e^{2 \beta p_{0}}-1} \theta\left(-p_{x}\right) \delta\left(p_{y}\right) \delta\left(p_{z}\right),
$$

This correctly reproduces $\left\langle\hat{T}_{b}^{a}\right\rangle$ as well as $\left\langle N^{a}\right\rangle$ which we shall compute below.

\section{E. Particle four-current $\left\langle\hat{N}_{\mu}(x)\right\rangle$}

In the kinetic theory, the first moment with respect to the four-momentum $p_{\mu}$ of the distribution function $\mathcal{W}(x, p)$ gives the particle four-current. Specifically, we have

$$
\left\langle\hat{N}_{\mu}(x)\right\rangle=\int d^{4} p p_{\mu}\langle\hat{\mathcal{W}}(x, p)\rangle \quad \text { with } \quad p_{0} \in(0,+\infty)
$$

Accordingly, the particle number density and its current are

$$
n(x)=\left\langle\hat{N}^{0}(x)\right\rangle \text { and } N^{i}(x)=\left\langle\hat{N}^{i}(x)\right\rangle,
$$

where the hydrodynamical velocity $U^{\mu}$ has been chosen of the form $(1,0,0,0)$. We merely note that $U^{\mu}$ corresponds neither Eckart nor Landau-Lifshitz velocity as these have to be light-like for the scalar model we have been considering.

We now go over to the study of this local macroscopic observable far away and close to the black-hole horizon.

\section{Far-horizon region}

Substituting $\mathcal{W}_{0}(x, p)$ given in Eq. (40) in the formula (52), we obtain

$$
n_{0}(x)=N_{0}^{r}(x)=\frac{1}{4 \pi R^{2}} \int \frac{d p_{0}}{2 \pi} \frac{\Gamma_{p_{0}}}{e^{\beta p_{0}}-1}+\mathrm{O}\left(\frac{r_{H}^{2} T_{H}^{4}}{R^{3}}\right),
$$


whereas $N_{0}^{\theta}(x)=N_{0}^{\phi}(x)=0$ identically. Note that this result can also be obtained with the aid of the effective one-particle distribution $f_{\text {eff, } 0}(x, p)$. It should also be emphasised that $n_{0}(x)$ originates from the monopole-like term of $\mathcal{W}_{0}(x, p)$, whereas $N_{0}^{r}$ comes from the dipole-like term in the Wigner distribution.

The number density as well as its current are positive, i.e. there is a positive radial particle flux from the black hole. To better understand what this means we go over to the region near the horizon.

\section{Near-horizon region}

Substituting $\mathcal{W}_{1}(x, p)$ in the definition of the particle four-current, we obtain

$$
n_{1}(x)=-N_{1}^{x}(x) \approx-\frac{1}{\pi r_{H}^{2}} \int \frac{d p_{0}}{2 \pi} \frac{\Gamma_{p_{0}}}{e^{2 \beta p_{0}}-1},
$$

while $N_{1}^{y}(x)$ and $N_{1}^{z}(x)$ are zero. ${ }^{5}$ This result implies that the density number of particles is negative at $R \sim r_{H}$, whereas its current is positive. The physical interpretation of $n_{1}(x)<0$ in terms of particles is here problematic as the number of particles per cubic centimetre cannot make any physical sense whenever negative.

One of the possible explanation of this result might be that quantum kinetic theory cannot adequately describe local physics near the horizon. Although the Wigner distribution $\mathcal{W}_{1}(x, p)$ properly reproduces the evaporation effect of black holes, the particle density may not have physical sense as the notion of particle may not be well-defined at $R \sim r_{H}$. On the other hand, it seems that the Wigner's concept of particle should be applicable in any local Minkowski frame, otherwise it would be unnatural to assume that this concept holds in local (approximately) Minkowski frame on earth only. If the Wigner's particle turns out to be physically realised at $R \sim r_{H}$, then $n_{1}(x)<0$ has to be physically understood.

Taking into account that there is no necessarily probabilistic interpretation of the Wigner distribution for quantum systems [6], it seems that there is still a physically non-excludable way of understanding $n_{1}(x)<0$. Specifically, one might think about $n_{1}(x)$ as a number of the field modes per cubic centimetre relative to its number density in local Minkowski frame. If so, then $n_{1}(x)<0$ would mean that the number of the field modes is smaller with respect to the flat case near the horizon. ${ }^{6}$ As a consequence, $n_{1}(x)=-N_{1}^{x}(x)<0$ should then imply the mode number decreases when one approaches the horizon. If one also associates a positive energy $p_{0}$ with each mode, one can then understand $\left\langle\hat{T}_{t_{F}}^{t_{F}}\right\rangle<0$ as the

\footnotetext{
${ }^{5}$ We have suppressed the index " $F$ " in the Fermi coordinates for the sake of transparency of the formulas. This should not cause any confusions as we employ all the time local inertial coordinates in both regions.

6 Note that if we consider a one-cubic-meter-size box with the gas of scalar particles of temperature $T>T_{H}$, then $n_{1}(x)$ will be positive within the volume of this box. We explain below why $T$ must actually be much bigger than $T_{H}$, i.e. $T \gg T_{H}$, in order for this set-up to make physical sense.
} 
total mode energy density relative to their total energy density in the absence of the black hole. In other words, this picture seems to fit well the near-horizon behaviour of the stress tensor $\left\langle\hat{T}_{b}^{a}\right\rangle$ following from $\mathcal{W}_{1}(x, p)$.

This manner of interpreting $n_{1}(x)<0$ as well as $\left\langle\hat{T}_{t_{F}}^{t_{F}}\right\rangle<0$ is mostly motivated by the physical understanding of the Casimir effect. This viewpoint is also consistent with our previous insights [9]. We come back to this issue below.

Comparing the behaviour of the particle four-current at $R \sim r_{H}$ and $R \gg r_{H}$, we find that $n(x)$ changes its sign at a certain distance $R_{c}$ away from the event horizon. We expect that it is of the order of $3 M$, i.e. at the distance where the energy flux changes its sign (e.g.,

see [16 20]). In one of our forthcoming papers, we shall try to carefully study this region with the help of the particle four-current.

\section{F. Entropy four-current $\left\langle\hat{S}^{\mu}(x)\right\rangle$}

The macroscopic variables $\left\langle\hat{T}_{\nu}^{\mu}\right\rangle$ and $\left\langle\hat{N}^{\mu}\right\rangle$ at $R \gg r_{H}$ behave like those of a steady flux of the stellar wind of distance-independent temperature. Therefore, $s_{0}(x)$ coincides with the entropy density of that kind of the idealised stellar wind.

As shown above, this picture is inapplicable in the near-horizon region. Moreover, the entropy density $s_{1}(x)$ turns out to be imaginary. Specifically, its imaginary part is ambiguous and reads

$$
\operatorname{Im} s_{1}(x)=(\pi+2 \pi k) n_{1}(x) \text { with } \quad k \in \mathbb{Z} .
$$

We do not understand how it can be interpreted in terms of statistical properties of some normal many-particle system.

\section{CONCLUDING REMARKS}

\section{A. Scalar field splitting}

If we consider the fundamental field operator $\hat{\Phi}(x)$ in the local inertial frame in the far-horizon and near-horizon region, then we find that it possesses the following structure:

$$
\hat{\Phi}(x)=\hat{\Phi}_{M}(x)+\delta \hat{\Phi}(x) \text { with }\left[\hat{\Phi}_{M}(x), \delta \hat{\Phi}\left(x^{\prime}\right)\right]=0,
$$

where $\hat{\Phi}_{M}(x)$ is the field operator as if there is no black hole, whereas $\delta \hat{\Phi}(x)$ vanishes as $r_{H} / R$ in the asymptotically flat region $\left(R \gg r_{H}\right)$ and is of $\mathrm{O}(1)$ near the black-hole horizon $\left(R \sim r_{H}\right)$. The field operator $\hat{\Phi}(x)$ before the collapse can be split in a sum of two nonfundamental operators with non-intersecting supports, namely $\hat{\Phi}_{<}(x)$ and $\hat{\Phi}_{>}(x)$, such that $\hat{\Phi}_{<}(x)$ vanishes for the Finkelstein-Eddington time $v>v_{H}$, where $v_{H}$ corresponds to the moment when the event horizon forms, whereas $\hat{\Phi}_{>}(x)$ vanishes for $v<v_{H}$. One can further 
split $\hat{\Phi}_{<}(x)$ into $\hat{\Phi}_{c}(x)$ and $\hat{\Phi}_{b}(x)[1,21]$, such that $\hat{\Phi}_{c}(x)$ has a vanishing support outside of the black-hole horizon, whereas $\hat{\Phi}_{b}(x)$ vanishes inside the horizon. ${ }^{7}$

In terms of the non-fundamental operators $\hat{\Phi}_{>}(x)$ and $\hat{\Phi}_{b}(x)$ for $R>r_{H}$, we have

$$
\begin{aligned}
& \hat{\Phi}_{M}(x)=\hat{\Phi}_{>}(x) \quad \text { and } \quad \delta \hat{\Phi}(x)=\hat{\Phi}_{b}(x) \quad \text { for } \quad R \gg r_{H}, \\
& \hat{\Phi}_{M}(x)=\hat{\Phi}_{b}(x) \quad \text { and } \quad \delta \hat{\Phi}(x)=\hat{\Phi}_{>}(x) \quad \text { for } \quad R \sim r_{H} .
\end{aligned}
$$

Therefore, the operator $\hat{\Phi}_{b}(x)$ is as physically relevant as $\hat{\Phi}_{>}(x)$ and vice verse for having a proper singularity structure in the field propagator far away from as well as near to the event horizon. It implies, for instance, that it is not legitimate to omit $\hat{\Phi}_{>}(x)$ in the asymptotically flat region, contrary to the common practice. Precisely this part of $\hat{\Phi}(x)$ has been successfully exploiting in particle physics, but do not contribute to the covariant Wigner distribution $\mathcal{W}_{0}(x, p)$.

The crucial role is, however, played by $\hat{\Phi}_{b}(x)$ near the event horizon as this part of $\hat{\Phi}(x)$ provides the proper singularity in the 2-point function $W_{1}\left(x, x^{\prime}\right)$ and, hence, allows to have the Feynman propagator with its ordinary interpretation in particle physics. The Wigner distribution $\mathcal{W}_{1}(x, p)$ we have derived above is completely independent on $\hat{\Phi}_{b}(x)$.

The splitting (57) is of no physical sense in a local inertial frame falling in the blackhole geometry. Still, the field operator $\hat{\Phi}(x)$ as being fundamental and its Hilbert space representation makes physical sense all the way down to the black hole. This is contrary to the tacitly proposed idea to define a separate Hilbert space for each of the non-fundamental operators on the right-hand side of Eq. (57). This idea eventually leads to the conclusion that the far-horizon region has to be described by a thermal density matrix. We do not share this point of view as it is beyond of our current understanding of local quantum field theory and, actually, inconsistent with that by construction [9].

\section{B. Scalar field particles and Wigner distribution}

The scalar operator $\hat{\Phi}(x)$ acquires the rich physical meaning in QFT when one represents it as the sum of two non-Hermitian field operators, namely $\hat{\Phi}(x)=\hat{a}(x)+\hat{a}^{\dagger}(x)$. The operator $\hat{a}(x)$ is in turn defined through the equation

$$
\hat{a}(x)=\int \frac{d^{4} k}{(2 \pi)^{3}} \theta\left(k_{0}\right) \delta\left(k^{2}\right) \Phi_{\mathbf{k}}(x) \hat{a}_{\mathbf{k}}
$$

where $\Phi_{\mathbf{k}}(x)$ are the mode functions being positive-frequency solutions (with respect to $P_{0}$ of the local $^{8}$ Poincaré group) of the scalar field equation and satisfy the normalisation condition

${ }^{7}$ It should be noted that the modes $u_{i n}(x \mid l, m, \omega, 1)$ defined in [21] are associated with the operator $\hat{\Phi}_{<}(x)$, while $u_{i n}(x \mid l, m, \omega, 2)$ with $\hat{\Phi}_{>}(x)$. The modes $u_{\text {in }}(x \mid l, m, \omega, 1)$ can be further split into $u_{\text {out }}(x \mid l, m, \omega, 0)$ and $u_{\text {out }}(x \mid l, m, \omega, 1)$. These are related to $\hat{\Phi}_{c}(x)$ and $\hat{\Phi}_{b}(x)$, respectively.

${ }^{8}$ We find ourselves in a local (approximately) inertial frame on earth. Therefore, the Poincaré group in particle physics is local as well. Although the universe is not globally flat at macroscopic scales, the 
$\left(\Phi_{\mathbf{p}}, \Phi_{\mathbf{k}}\right)_{\mathrm{KG}}=\delta(\mathbf{p}-\mathbf{k})$. The vacuum $|\Omega\rangle$ is defined through the equation $\hat{a}_{\mathbf{k}}|\Omega\rangle=0$.

The one-particle state $|\mathbf{k}\rangle=\hat{a}_{\mathbf{k}}^{\dagger}|\Omega\rangle$ is not normalisable. The physical 1-particle state is defined through the covariant wave packet $h(x)$ :

$$
h(x)=\int \frac{d^{4} k}{(2 \pi)^{3}} \theta\left(k_{0}\right) \delta\left(k^{2}\right) h(k) \Phi_{\mathbf{k}}(x),
$$

where $h(k)$ is a square-integrable function. A localised particle state described by $h(k)$ is

$$
|h\rangle \equiv \hat{a}^{\dagger}(h)|\Omega\rangle \equiv\left(h^{*}, \hat{\Phi}\right)_{\mathrm{KG}}|\Omega\rangle=\int \frac{d^{4} k}{(2 \pi)^{3}} \theta\left(k_{0}\right) \delta\left(k^{2}\right) h(k)|\mathbf{k}\rangle,
$$

which is normalisable, i.e. $\langle h \mid h\rangle=1$, as having a finite support.

There are infinitely many ways of splitting the field operator $\hat{\Phi}(x)$ in the sum of nonHermitian operators. This is a direct consequence of the linearity of the field equation. The proposal was to choose different mode functions for different coordinate frames. This usually implies that it is meaningful to have different notions of particles in different frames. This resulted eventually in a belief that "quantum mechanics is observer-dependent". We do not share this point of view as it leads to the various paradoxical/unphysical conclusions. Recently, we have proposed another principle which is conservative in its spirit and based on the idea of the equivalence principle [4]. To make it short, the mode functions $\Phi_{\mathbf{p}}(x)$ defining a physical, observer-independent notion of particles are those which acquire the Minkowski structure, namely

$$
\Phi_{\mathbf{k}}(x) \sim \exp \left(-i k_{\mu} x^{\mu}\right)
$$

in a local inertial frame defined at each point of spacetime. This makes sense only in spacetime regions with not too strong gravity. The main argument in favour of this definition is that we have been doing this all the time on earth to predict and describe various scattering processes in the particle colliders.

Indeed, a well-tested notion of the particle is associated with the unitary, irreducible representations of the Poincaré group $\mathcal{P}_{+}^{\uparrow}$. This idea was proposed long ago by Wigner (e.g., see [22]). The Poincaré group forms here the isometry of local Minkowski frame only, as the universe is globally non-flat. This is a basic idea behind of our proposal of relating the well-tested notion of the particle in Minkowski space with its definition in curved spacetime. Note that the particle in a non-inertial frame is described by an appropriate covariant wave packet of non-vanishing acceleration.

Once we have defined a wave packet, we have the 1-particle state carrying information about the particle. The wave packet is characterised by its non-vanishing support. Normally, it should correspond to the size of the particle. In our case, this is given by the de Broglie

Minkowski-space approximation is fully enough to successfully describe scattering processes in the particle colliders. 
wavelength $\lambda_{\mathbf{k}}$ of the scalar particle. Therefore, the correction to the right-hand side of (62) near horizon must be suppressed by a factor of $\left(\lambda_{\mathbf{k}} / r_{H}\right)^{2} \ll 1$, otherwise there is no well-defined notion of the particle in the Wigner sense. ${ }^{9}$ This is indeed the case.

Thus, we cannot relate the Wigner distribution $\mathcal{W}(x, p)$ we have found above to the real particles as this originates from the suppressed correction to the right-hand side of (62).

\section{Negative particle density and quantum noise}

The main idea of defining $\mathcal{W}(x, p)$ in $\mathrm{QFT}$ is to have a distribution function derived from the first principles with the aid of which one can determine the local macroscopic state variables characterising many-particle systems [6]. Indeed, we have seen that the Wigner distribution $\mathcal{W}(x, p)$ can be used to compute the stress tensor $\left\langle\hat{T}_{\nu}^{\mu}\right\rangle$ and the particle four-flow $\left\langle\hat{N}^{\mu}\right\rangle$ as its second and first moment with respect to the four-momentum $p_{\mu}$, respectively.

We have shown that the particle four-current $N^{\mu}=\left(n_{0}, N_{0}^{r}, 0,0\right)$ can make physical sense as a steady outward particle flow in the asymptotically flat region. This is in full agreement with [1]. However, this interpretation of $N^{\mu}=\left(n_{1}, N_{1}^{x}, 0,0\right)$ is inapplicable in the nearhorizon region, because $n_{1}<0$ cannot be possible for the real particles and qualitatively differs from a behaviour of a normal relativistic gas [24].

As pointed out above, $\mathcal{W}(x, p)$ comes in the present set-up from the correction to the leading term of the mode functions (see Eq. (62) ). This correction plays a sub-leading role in the definition of the particle creation operator $\hat{a}^{\dagger}(h)$ of the wave function $h(x)$, but the leading role for $\mathcal{W}(x, p)$ to be non-trivial. Therefore, we think that $\mathcal{W}(x, p)$ with its moments are entirely due to the quantum fluctuations described by that correction which is in turn induced by the presence of the black hole. The number of the modes characterising these fluctuations turns out to be smaller at $R<R_{c}$ than that in the absence of the black hole. As a consequence, its relative number density and energy density are negative.

If so, a noval property of the quantum fluctuations would be their "ability" to transfer energy (through gravity playing a role of the "working body"). This does not seem to be a completely speculative idea bearing in mind a lab set-up we described in [25]. Specifically, one can compute the vacuum energy density in two cavities separated by an extra metallic plate in the Casimir set-up when this plate is in the middle and when it is shifted in a way the dynamical Casimir effect is negligible. Comparing the total vacuum energy density after and before the shift, one finds that its absolute value has increased. Thus, the negative vacuum energy has been partially redistributed between the cavities and partially dissipated in the middle plate by heating it up. The middle plate in this process plays a role of the working body.

\footnotetext{
${ }_{9}^{9}$ It seems that we are in agreement at this point with [23] (see paragraph 3 on p. 2).
} 


\section{ACKNOWLEDGMENTS}

It is a pleasure to thank Frans Klinkhamer and José Queiruga for discussions.

\section{Appendix A: Vacuum expectation value of stress tensor $\hat{T}_{\mu \nu}(x)$}

The stress tensor $T_{\mu \nu}(x)$ of the (classical) massless scalar field $\Phi(x)$ conformally coupled to gravity is given by

$$
T_{\mu \nu}=\frac{2}{3} \nabla_{\mu} \Phi \nabla_{\nu} \Phi-\frac{1}{6} g_{\mu \nu} \nabla_{\lambda} \Phi \nabla^{\lambda} \Phi-\frac{1}{3} \Phi \nabla_{\mu} \nabla_{\nu} \Phi
$$

Employing the point-splitting technique to get the renormalised value of the radial energy flux, we obtain

$$
\left\langle\hat{T}_{t r}\right\rangle=\frac{1}{3} \lim _{x^{\prime} \rightarrow x}\left[\left(\partial_{t} \partial_{r^{\prime}}+\partial_{t^{\prime}} \partial_{r}\right)-\frac{1}{2}\left(\nabla_{t} \nabla_{r}+\nabla_{t^{\prime}} \nabla_{r^{\prime}}\right)\right] W\left(x, x^{\prime}\right)= \pm \frac{r_{H}^{2}}{r^{2} f} \int \frac{d \omega \omega}{(4 \pi)^{2}} b_{\omega}(
$$

for both the far-horizon and near-horizon region. The $\Delta r_{\star}$-term in the bi-scalar $\chi_{\omega}\left(\mathbf{x}, \mathbf{x}^{\prime}\right)$ is crucial for having non-vanishing radial energy flux.

It is straightforward to further show that

$$
\begin{aligned}
\left\langle\left(\partial_{t} \hat{\Phi}\right)^{2}\right\rangle & =+\frac{g_{t t}}{f} \int \frac{\omega d \omega}{(4 \pi)^{2}} \bar{\chi}_{\omega}(\mathbf{x}, \mathbf{x}) \\
\left\langle\left(\partial_{i} \hat{\Phi}\right)^{2}\right\rangle & =-\frac{g_{i i}}{3 f} \int \frac{\omega d \omega}{(4 \pi)^{2}}\left[1+\frac{\bar{R}_{i}^{i}}{2 \omega^{2} f}\right] \bar{\chi}_{\omega}(\mathbf{x}, \mathbf{x})+\int \frac{d \omega}{(4 \pi)^{2} \omega} \lim _{\mathbf{x}^{\prime} \rightarrow \mathbf{x}} \partial_{(i} \partial_{\left.i^{\prime}\right)} \bar{\chi}_{\omega}\left(\mathbf{x}, \mathbf{x}^{\prime}\right),
\end{aligned}
$$

where there is no summation over $i=\{r, \theta, \phi\}$ in the second line, and we have introduced a new bi-scalar as follows

$$
\bar{\chi}_{\omega}\left(\mathbf{x}, \mathbf{x}^{\prime}\right) \equiv \frac{\chi_{\omega}\left(\mathbf{x}, \mathbf{x}^{\prime}\right)}{\left(f(r) f\left(r^{\prime}\right)\right)^{\frac{1}{2}}}
$$

and

$$
\begin{aligned}
& \left\langle\hat{\Phi} \hat{\Phi}_{; t t}\right\rangle=-\frac{g_{t t}}{f} \int \frac{\omega d \omega}{(4 \pi)^{2}} \bar{\chi}_{\omega}(\mathbf{x}, \mathbf{x})+\frac{1}{2} \int \frac{d \omega}{(4 \pi)^{2} \omega} \lim _{r^{\prime} \rightarrow r}\left[\nabla_{t}^{2}+\nabla_{t^{\prime}}^{2}\right] \bar{\chi}_{\omega}\left(r, r^{\prime}\right) \\
& \left\langle\hat{\Phi} \hat{\Phi}_{; i i}\right\rangle=\frac{g_{i i}}{3 f} \int \frac{\omega d \omega}{(4 \pi)^{2}}\left[1+\frac{\bar{R}_{i}^{i}}{2 \omega^{2} f}\right] \bar{\chi}_{\omega}(\mathbf{x}, \mathbf{x})+\frac{1}{2} \int \frac{d \omega}{(4 \pi)^{2} \omega} \lim _{\mathbf{x}^{\prime} \rightarrow \mathbf{x}}\left[\nabla_{i}^{2}+\nabla_{i^{\prime}}^{2}\right] \bar{\chi}_{\omega}\left(\mathbf{x}, \mathbf{x}^{\prime}\right) .
\end{aligned}
$$

The vacuum expectation value of the trace of the non-renormalised stress tensor must vanish:

$$
\left\langle\hat{T}_{\mu}^{\mu}\right\rangle=-\frac{1}{3}\langle\hat{\Phi} \square \hat{\Phi}\rangle=-\frac{1}{3 f^{2}} \int_{\mathbf{R}} \frac{d \omega}{(4 \pi)^{2} \omega} \lim _{\mathbf{x}^{\prime} \rightarrow \mathbf{x}} \square \chi_{\omega}\left(\mathbf{x}, \mathbf{x}^{\prime}\right)=0 .
$$




\section{a. Far-horizon region}

Substituting the bi-scalar $\chi_{\omega}\left(\mathbf{x}, \mathbf{x}^{\prime}\right)$ given in Eq. (11) with Eq. (12), we obtain

$$
\left\langle\hat{T}_{\nu}^{\mu}\right\rangle=\frac{1}{f^{2}} \int \frac{d \omega \omega a_{\omega}}{(4 \pi)^{2}}\left[\begin{array}{cc}
1 & 0 \\
0 & -\frac{1}{3} \cdot 1_{3 \times 3}
\end{array}\right]+\frac{1}{f R^{2}} \int \frac{d \omega \omega b_{\omega}}{(4 \pi)^{2}}\left[\begin{array}{ccc}
+1 & +1 & 0 \\
-1 & -1 & 0 \\
0 & 0 & 0_{2 \times 2}
\end{array}\right]+\mathrm{O}\left(\frac{1}{R^{5}}\right)(
$$

in the far-horizon region, i.e. for $R \gg r_{H}$.

\section{b. Near-horizon region}

In the near-horizon region, i.e. $R \sim r_{H}$, the non-renormalised stress-energy tensor reads

$$
\begin{aligned}
\left\langle\hat{T}_{\nu}^{\mu}\right\rangle=\frac{1}{f^{2}} \int \frac{d \omega \omega a_{\omega}}{(4 \pi)^{2}} & {\left[\begin{array}{cc}
1 & 0 \\
0 & -\frac{1}{3} \cdot 1_{3 \times 3}
\end{array}\right]+\int \frac{d \omega \omega b_{\omega}}{(4 \pi)^{2} r_{H}^{2}}\left[\begin{array}{ccc}
+\frac{1}{f} & -1 & 0 \\
+\frac{1}{f^{2}} & -\frac{1}{f} & 0 \\
0 & 0 & 0_{2 \times 2}
\end{array}\right] } \\
& -\frac{1}{3 r_{H}^{4}} \int \frac{d \omega b_{\omega}}{(4 \pi)^{2} \omega}\left[\begin{array}{cc}
+1_{2 \times 2} & 0 \\
0 & -1_{2 \times 2}
\end{array}\right] \times\left(1-\frac{11}{2} f+\frac{35}{2} f^{2}+\mathrm{O}\left(f^{3}\right)\right)
\end{aligned}
$$

for $\alpha_{\omega}\left(r, r^{\prime}\right)$ and $\beta_{\omega}\left(r, r^{\prime}\right)$ given in (12).

The matrix structure of the third term in Eq. (A8) is of the crucial importance, because it guarantees that this term is also finite on the horizon in the Fermi frame. It should be noted that the extra corrections to $\alpha_{\omega}\left(r, r^{\prime}\right)$ and $\beta_{\omega}\left(r, r^{\prime}\right)$ also contribute to this term to the leading order changing its numerical value and the sign as follows from the numerical results of [26]. This contribution to the stress tensor does not change its value and structure when rewritten in the Fermi frame like the Hartle-Hawking part (given in Eq. (45)).

The terms vanishing as $f(R)$ in the Schwarzschild frame also contribute in the Fermi frame. It implies that the difference $\left\langle\Delta \hat{T}_{b}^{a}\right\rangle \equiv\left\langle\hat{T}_{b}^{a}\right\rangle-\left\langle\hat{T}_{b}^{a}\right\rangle_{\mathrm{HH}}$ in the Fermi frame is actually given by

$$
\left\langle\Delta \hat{T}_{b}^{a}\right\rangle \approx-\frac{L}{16 \pi r_{H}^{2}}\left[\begin{array}{ccc}
+1 & -1 & 0 \\
+1 & -1 & 0 \\
0 & 0 & 0_{2 \times 2}
\end{array}\right]-\gamma_{1}\left[\begin{array}{cc}
+1_{2 \times 2} & 0 \\
0 & -1_{2 \times 2}
\end{array}\right]-\gamma_{2}\left[\begin{array}{ccc}
+1 & +1 & 0 \\
-1 & -1 & 0 \\
0 & 0 & 0_{2 \times 2}
\end{array}\right](
$$

near the event horizon with

$$
\begin{aligned}
\gamma_{1} & =\sum_{l=0}^{+\infty} \int \frac{d x}{4 \pi x} \frac{(2 l+1)\left|B_{\omega l}\right|^{2}}{e^{8 \pi x}-1} \frac{l(l+1)\left(1+24 x^{2}\right)+8 x^{2}}{6 \pi r_{H}^{4}\left(1+16 x^{2}\right)} \\
\gamma_{2} & =\sum_{l=0}^{+\infty} \int \frac{d x}{4 \pi x} \frac{(2 l+1)\left|B_{\omega l}\right|^{2}}{e^{8 \pi x}-1} \frac{2 l(l+1)\left(1+40 x^{2}\right)+3[l(l+1)]^{2}\left(1+8 x^{2}\right)+72 x^{2}}{12 \pi r_{H}^{4}\left(1+20 x^{2}+64 x^{4}\right)}
\end{aligned}
$$

where $x \equiv \omega M$, with the numerical values $\gamma_{1} \approx 1.25 \times 10^{-6} / M^{4}$ (in agreement with [26]) and $\gamma_{2} \approx 4.45 \times 10^{-6} / M^{4}$. It should be noted that $L / 16 \pi r_{H}^{2} \approx 9.25 \times 10^{-8} / M^{4}$ which is much 
smaller than $\gamma_{2}$. Still, the decrease of the black-hole mass $M$ is entirely due to $L$, namely $\dot{M}=-L$, where dot stands for the differentiation with respect to the Schwarzschild time coordinate. This means that the last term in Eq. (A9) is geometrical and it might be that one should throw away this from the solution of the field equation. Note that this term vanishes as $f(R)$ in the near-horizon region in the Schwarzschild frame.

The last two terms in (A9) cannot be described by any one-particle distribution $\tilde{f}_{\text {eff, }, 1}(\mathbf{p})$, because it must satisfy the condition

$$
\int \frac{d^{3} \mathbf{p}}{|\mathbf{p}|} \tilde{f}_{\mathrm{eff}, 1}(\mathbf{p})=0 \text {. }
$$

This follows from

$$
\Delta \tilde{W}_{1}\left(x, x^{\prime}\right)=\frac{1}{2}\left(\left(\gamma_{1}+\gamma_{2}\right) \Delta t^{2}-2 \gamma_{2} \Delta t \Delta x-\left(\gamma_{1}-\gamma_{2}\right) \Delta x^{2}+\gamma_{1} \Delta y^{2}+\gamma_{1} \Delta z^{2}\right),
$$

which vanishes in the coincidence limit, i.e. $x^{\prime} \rightarrow x$. Note that $\square \Delta \tilde{W}\left(x, x^{\prime}\right)=0$ exactly holds and $\Delta \tilde{W}_{1}\left(x, x^{\prime}\right)$ is locally suppressed as $\left(\Delta x / r_{H}\right)^{2}$ with respect to $\Delta W_{1}\left(x, x^{\prime}\right)$ and as $\left(\Delta x / r_{H}\right)^{4}$ with respect to $W_{M}\left(x, x^{\prime}\right) \approx W_{\mathrm{HH}}\left(x, x^{\prime}\right)$.

[1] S.W. Hawking, "Black hole explosions?", Nature 248, 30 (1974); "Particle creation by black holes", Commun. Math. Phys. 43, 199 (1975).

[2] D.N. Page, "Thermal stress tensors in static Einstein spaces", Phys. Rev. D25, 1499 (1982).

[3] S. Emelyanov, "Effective photon mass from black-hole formation", Nucl. Phys. B919, 110 (2017), arXiv:hep-th/1603.01148.

[4] S. Emelyanov, "Near-horizon physics of evaporating black holes", arXiv:hep-th/1608.05318.

[5] P. Candelas, "Vacuum polarization in Schwarzschild spacetime", Phys. Rev. D21, 2185 (1980).

[6] S.R. de Groot, W.A. van Leeuwen, C.G. van Weert, Relativistic Kinetic Theory, (NorthHolland, 1980).

[7] S.M. Christensen, S.A. Fulling, "Trace anomalies and the Hawking effect", Phys. Rev. D15, 2088 (1977).

[8] F.K. Manasse, S.W. Misner, "Fermi normal coordinates and some basic concepts in differential geometry", J. Math. Phys., 4, 735 (1963).

[9] S. Emelyanov, "Can gravitational collapse and black-hole evaporation be a unitary process after all?", arXiv:hep-th/1507.03025.

[10] S. Emelyanov, "Low-energy electromagnetic radiation as an indirect probe of black-hole evaporation", Nucl. Phys. B913, 318 (2016), arXiv:hep-th/1602.01475.

[11] C. Cercignani, G.M. Kremer, The relativistic Boltzmann equation: Theory and Applications, (Birkhäuser Verlag, Basel, 2002).

[12] V. Moretti, "Comments on the stress energy tensor operator in curved space-time", Commun. Math. Phys. 232, 189 (2003), arXiv:gr-qc/0109048. 
[13] Y. Décanini, A. Folacci, "Hadamard renormalization of the stress-energy tensor for a quantized scalar field in a general spacetime of arbitrary dimension", Phys. Rev. D78, 044025 (2008), arXiv:gr-qc/0512118.

[14] K.W. Howard, P. Candelas, "Quantum stress tensor in Schwarzschild space-time", Phys. Rev. Lett. 53, 403 (1984).

[15] W.G. Unruh, "Origin of the particles in black-hole evaporation", Phys. Rev. D15, 365 (1977).

[16] S.B. Giddings, "Hawking radiation, the Stefan-Boltzmann law, and unitarization", Phys. Lett. B754, 39 (2016), arXiv:hep-th/1511.08221.

[17] S.B. Giddings, "Observational strong gravity and quantum black hole structure", Int. J. Mod. Phys. D25, 1644014 (2016), arXiv:gr-qc/1605.05341.

[18] S. Hod, "Hawking radiation and the Stefan-Boltzmann law: The effective radius of the blackhole quantum atmosphere", Phys. Lett. B757, 121 (2016), arXiv:qr-gc/1607.02510.

[19] R. Dey, S. Liberati, D. Pranzetti, "The black hole quantum atmosphere", arXiv:grqc/1701.06161.

[20] S.B. Giddings, "Astronomical tests for quantum black hole structure", Nature Astronomy 1, 0067 (2017), arXiv:gr-qc/1703.03387.

[21] B. DeWitt, The global approach to quantum field theory (V2, Oxford University Press, 2003).

[22] R. Haag, Local quantum physics. Fields, Particles, Algebras (Springer-Verlag, 1996).

[23] J.M. Bardeen, "Black hole evaporation without an event horizon", arXiv:gr-qc/1406.4098.

[24] G.M. Kremer, "Relativistic gas in a Schwarzschild metric", J. Stat. Mech. 1304, P04016 (2013), arXiv:gr-qc/1212.5573.

[25] S. Emelyanov, "Quantum electromagnetic phenomena far from small evaporating black holes", in Proceedings, New Frontiers in Black Hole Astrophysics (IAU Symposium No. 324, 2016), arXiv:hep-th/1612.07637.

[26] T. Elster, "Vacuum polarization near a black hole creating particles", Phys. Lett. A94, 205 (1983). 